\title{
Simple and fast quantification of DNA damage by real-time PCR, and its application to nuclear and mitochondrial DNA from multiple tissues of aging zebrafish
}

\author{
Shusen Zhu and James A. Coffman * (1)
}

\begin{abstract}
We describe a real-time (rt) PCR-based method of quantifying DNA damage, adapted from the long-run rtPCR method of DNA damage quantification (LORD-Q) developed by Lehle et al. (Nucleic Acids Res 42(6):e41, 2014). We show that semi-long run rtPCR, which generates amplicons half the length of those generated in LORD-Q, provides equivalent sensitivity for detecting low lesion frequencies, and better sensitivity for detecting high frequencies. The smaller amplicon size greatly facilitates PCR optimization and allows greater flexibility in the use of detection dyes, and a modified data analysis method simplifies the calculation of lesion frequency. The method was used to measure DNA damage in the nuclear and mitochondrial genomes of different tissues in zebrafish of different ages. We find that nuclear DNA damage generally increases with age, and that the amount of mitochondrial DNA damage varies substantially between tissues, increasing with age in liver and brain but not in heart or skeletal muscle, the latter having the highest levels of damage irrespective of age.
\end{abstract}

Keywords: DNA damage, Quantitative PCR, Nuclear, Mitochondrial, Aging, Zebrafish

\section{Background}

The information encoded in the nucleotide sequence of genomic DNA is essential for biological function, and cells employ numerous mechanisms to repair damaged DNA and maintain genomic integrity. Nonetheless, the frequency of genotoxic insults can overcome a cell's capacity for DNA repair, leading to the accumulation of DNA damage that can in turn lead to cell death or senescence, or transformation to nonfunctional or pathological cellular phenotypes. The question of how much damage is present in the DNA of a cell, tissue, or organism at different stages of a life cycle or in different environmental or physiological circumstances is central to various fields of biomedicine, including toxicology, oncology, and gerontology;

*Correspondence: jcoffman@mdibl.org

MDI Biological Laboratory, Salisbury Cove, ME, USA hence, robust, sensitive, and user-friendly methods of quantifying DNA damage are widely called for in biomedical science.

Since lesions that disrupt DNA integrity interfere with DNA replication, DNA damage can be measured by polymerase chain reaction (PCR). Quantitative PCR (qPCR) methods of measuring DNA damage make use of the fact that replication efficiency is proportional to the average frequency of lesions within a defined region, and hence the length of that region [1]. Generally these methods work by comparing the ratios of long and short amplicons obtained from different samples, the latter providing a baseline (virtually damage-free) reference. Originally this approach made use of end-point PCR [2]. More recently a real-time PCR method of using longrun real-time (rt) PCR for DNA damage quantification (LORD-Q) was described which allowed sensitive and robust detection of the number of lesions (including double-stranded breaks, abasic sites, thymine dimers, and 
5-hydroxymethyl $\mathrm{dC}$ ) within a defined sequence [3]. As originally described this method requires amplification of relatively long sequences, $\sim 3-4 \mathrm{~kb}$ in length.

Although PCR is straightforward in theory, in practice it often requires extensive optimization to maximize efficiency and specificity, and the ease of optimization decreases with increasing amplicon length. We therefore asked whether the LORD-Q method could be adapted to work with shorter amplicons. We find that semi-long run (SLR) amplicons of $\sim 1.5-2 \mathrm{~kb}$ provide sensitivity equivalent to that provided by LORD-Q for detection of low-frequency damage, and better sensitivity for high-frequency damage. Shorter amplicons make PCR conditions less stringent and therefore easier to optimize. To demonstrate the utility of this method, we use it to quantify DNA damage in the nuclear and mitochondrial genomes from multiple tissues of zebrafish of different ages. Unlike most studies in the past that that made such measurements following differential isolation of the mtDNA and nDNA, in our analysis the mtDNA and nDNA were co-isolated, allowing their direct comparison. Our results show that DNA damage varies between tissues, is on average higher in mitochondrial DNA than in nuclear DNA, and generally increases with age.

\section{Methods}

\section{DNA isolation and purification}

Total DNA was purified from zebrafish tissues using E.Z.N.A. tissue DNA kit (OMEGA, USA). DNA quantity and purity were determined by spectrometry using a Nanodrop 1000. The purified DNA (A260/A280 $\geq 1.8$ ) was stored at $4{ }^{\circ} \mathrm{C}$.

\section{Primers for the real-time PCR}

For comparison of the sensitivity and accuracy of long run (LR) and semi-long run (SLR) PCR of nuclear DNA (nDNA) and mitochondrial DNA (mtDNA), and to examine the difference in the amount of damage in coding region and D-loop regions of mtDNA, we designed 2 pairs of long-run primers for amplicons of 3.5-4 kb, 3 pairs of semi-long-run primers for amplicons of $1.6-2 \mathrm{~kb}$, and 2 pairs of short run primers for amplicons of $55 \mathrm{bp}$ which served as internal undamaged references for normalization (Table 1). All three pairs of primers for nDNA are located in the zebrafish (Danio rerio) aryl hydrocarbon receptor2 (AHR2) gene, and the four pairs of primers for zebrafish mtDNA cover different regions. The primers were designed using program $\mathrm{NCBI}$ /primer-BLAST (http://www.ncbi.nlm.nih.gov/tools/primer-blast/) and synthesized by Integrated DNA Technologies (IDT, USA) with standard desalting.

\section{Enzymatic digestion of isolated DNA}

For quantification of defined lesion frequencies, $5 \mu \mathrm{g}$ of total DNA isolated from 7 month-old zebrafish brains was subjected to enzymatic digestion with the restriction endonuclease PvuII (for SLR PCR), identified as single cutter for both the targeted nDNA (1637 bp of AHR2 gene) and mtDNA (1978 bp), or NdeI (for LR PCR), identified as single cutter for both the targeted nDNA (3502 bp of AHR2 gene) and mtDNA (3669 bp), using the NEBcutter V2.0 software (http://nc2.neb.com/ NEBcutter2). Digestion was carried out at $37{ }^{\circ} \mathrm{C}$ for $2 \mathrm{~h}$ in a $100-\mu \mathrm{l}$ volume using 50 units $P v u \mathrm{II}$ and $N d e \mathrm{I}$ (NEB) respectively. For the analysis of DNA-lesion frequency, digested and undigested DNA was diluted and mixed at

Table 1 Primers and targets

\begin{tabular}{|c|c|c|c|}
\hline Primer & Sequence & Amplicon (bp) & Target \\
\hline AHRLF & GTCCTTGCAGGTTGGCAAATGG & 3502 & AHR2 \\
\hline AHRLR & GACCTTGTCTGGTTTTCATCCC & & \\
\hline AHRSLF & TCATCCTGTTATCCACCACACTGTTG & 1637 & AHR2 \\
\hline AHRSLR & TGGTTCTTGGCTACACTGAGATTGAG & & \\
\hline AHRRF & CCAAGGTCCGACATAACTCACTTCTG & 55 & AHR2 \\
\hline AHRRR & GACATGATGTACTGTGCTGACAACCA & & \\
\hline mtLF & TTAAAGCCCCGAATCCAGGTGAGC & 3669 & mtDNA coding region \\
\hline$m t L R$ & TTAGGGGTAGTGAGTTTTGGGTC & & \\
\hline $\mathrm{mtSLF}$ & GGATTCCAAGACGCAGCATCACCTG & 1978 & mtDNA coding region \\
\hline $\mathrm{mtSLR}$ & GGAGCGGCACTTCAAATGGGTCAAG & & \\
\hline mtDLPF & CCTTACACGATTCTTCGCATTCCAC & 1939 & mtDNA D-loop region \\
\hline$m t D L P R$ & GGCTTGGCTAGGCGTCTTGG & & \\
\hline mtRF & CGAGGAGCAGGTATCAGGCACA & 55 & mtDNA coding region \\
\hline $\mathrm{mtRR}$ & GTGGCTTGGCTAGGCGTCTTG & & \\
\hline
\end{tabular}


different ratios (Additional file 1: Table S1) prior to realtime PCR quantification.

\section{DNA irradiation}

$1.5 \mu \mathrm{l}$ of purified DNA from brains of 5-month-old zebrafish was loaded on a piece of parafilm and exposed to ultraviolet $C$ radiation (UVC) at dose of $0.5,1,2,5,10$, 20 and $50 \mathrm{~mJ} / \mathrm{cm}^{2}$ in a UV crosslinker (UVP HL-2000 HybriLinker, USA). Irradiated DNA was then diluted to $16 \mathrm{ng} / \mu \mathrm{l}$ for $\mathrm{nDNA}$ and $2 \mathrm{ng} / \mu \mathrm{l}$ for mtDNA damage assay.

\section{Real-time PCR}

Real-time PCR (rtPCR) for DNA damage quantification was carried out in a LightCycler 480 II system (Roche) in 96-well plates. The PCR conditions were optimized for the different primers and different targets to get specific products which were confirmed by melting curve analysis and agarose gels (Fig. 1). The PCR amplification was monitored by real-time measurement of the intercalation of the saturating fluorescent dye into dsDNA. $4 \mathrm{ng}$ of nDNA sample or $1 \mathrm{ng}$ of mtDNA sample was applied in $10 \mu \mathrm{l}$ of total reaction volume. Standard curves were created by serial dilution of the undamaged control with concentration of $16,4,1,0.25 \mathrm{ng}$ of nDNA and 4, 1, 0.25, $0.0625 \mathrm{ng}$ of mtDNA for quantification of all targets of different sizes. Total DNA from brains of 5-month-old zebrafish (found to have the lowest amount of DNA damage; see Fig. 4) served as undamaged reference. Each sample was assayed in triplicate. For LR and SLR realtime PCR, Platinum Pfx DNA polymerase (ThermoFisher Scientific, USA) was employed and the reactions containing 1X fluorescent dye were performed in 96-well clear plates. Resolight dye was used for all experiments

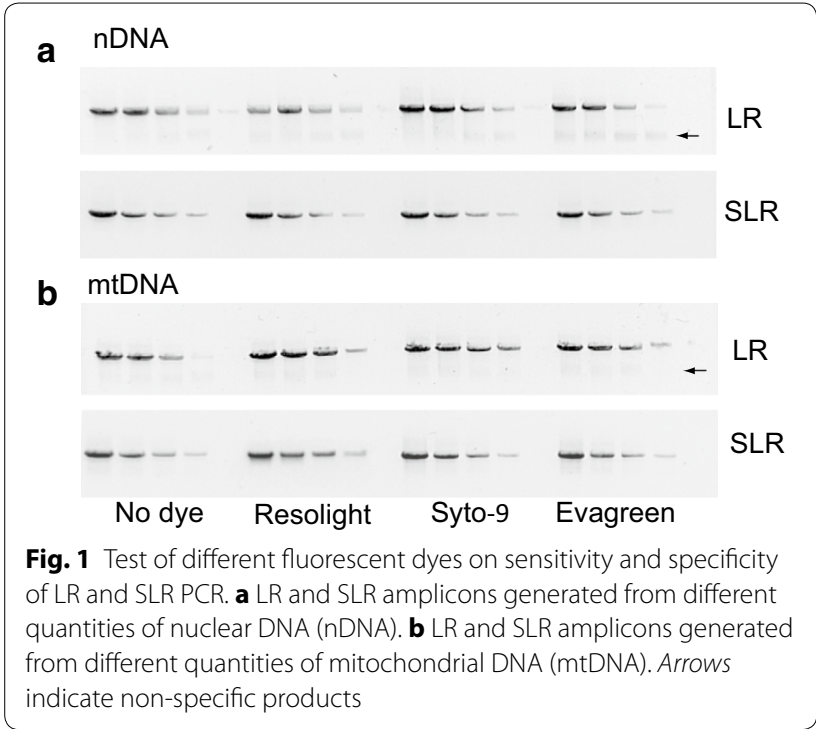

except that which compared the effect of different dyes on the specificity and efficiency of the PCR, which used 1X Resolight (Roche), $1.5 \mu \mathrm{M}$ SYTO-9 (ThermoFisher Scientific, USA) or 1X EvaGreen (Biotium, USA). For reference quantification (short run), PerfeCTa SYBR Green FastMix (Quanta BioSciences) was used, and the reactions were performed in 96-well white plates. In initial trials comparing Pfx DNA polymerase with hot start Taq polymerase, Pfx was found to be more efficient and more specific than hot start Taq.

\section{Data analysis}

Absolute quantification and second derivative maximum analysis methods were applied for all PCRs (LightCycler 480 Instrument Operator's Manual, v1.5, 2012). Sample and reference DNA were analyzed side-by-side. DNA damage is quantified by normalizing the concentration values of samples to the concentration values of the internal reference. The resulting values are converted to relative lesion frequencies per $10 \mathrm{~kb}$ DNA by application of the Poisson distribution (lesions/amplicon $=-\ln \left(\mathrm{A}_{\mathrm{t}} /\right.$ $\left.A_{0}\right)$ ), where $A_{t}$ represents the amplification (normalized concentration) of treated samples and $\mathrm{A}_{0}$ is the amplification of untreated or undamaged controls [1].

\section{Lesions per $10 \mathrm{~kb} D N A$}

$=\left(-\ln \left(A_{t} / A_{0}\right)\right) \times 10,000[\mathrm{bp}] /$ size of long fragment $[\mathrm{bp}]$

long fragment represents the LR or SLR amplicon.

Statistical analysis of intergroup differences was performed using one-way or two-way ANOVA and Student's t test.

\section{Results}

\section{Semi long-run PCR is more efficient than long-run PCR} and the products are more specific

We first used standard PCR to compare the specificity of the LR PCR (3-4 kb) used in LORD-Q to that of SLR PCR $(1.6-2 \mathrm{~kb})$, as assessed by the appearance of non-specific amplification products on a gel. This was done both without dye added to the reaction, and in the presence of a fluorescent intercalating dye such as is used in real-time PCR. The commonly used fluorescent dye SYBR Green inhibits synthesis of long PCR products, so LORD-Q instead uses the non-inhibitory dye Resolight [3]. We also tested two additional dyes reported to have low inhibitory effects, Syto- 9 and Evagreen, which are considerably less expensive than Resolight (340- and 11-fold, respectively). With both nDNA and mtDNA primer sets, SLR PCR yielded a single product in the presence of all three dyes (Fig. 1). In contrast, the LR PCRs generated nonspecific products visible as smears or non-specific bands following agarose gel electrophoresis (Fig. 1, arrows), indicating that all three dyes reduce the specificity of LR 
PCR, with Evagreen having the biggest negative effect. These results indicate that in the presence of intercalating dye SLR PCR is more specific than LR PCR.

\section{The sensitivity of the SLR real-time PCR based DNA damage assay is comparable to the LORD-Q method}

To compare the use of SLR versus LR PCR in a real-time PCR-based DNA damage assay, we digested DNA with restriction endonucleases predicted to make a single cut within the amplified mtDNA and nDNA fragments. The digested DNA was then mixed with undigested DNA at different ratios to generate a standard curve, and both SLR and LR real-time PCR were performed to measure the premade DNA damage. The expected lesion rates were calculated and plotted against the experimentally measured damage (Fig. 2; Additional file 1: Table S1). With both LR and SLR PCR, the measured lesion frequencies tracked the expected frequencies for both the mtDNA and nDNA, albeit more closely with SLR PCR with increasing levels of damage (Fig. 2), suggesting that the latter provides somewhat greater accuracy at higher levels of damage.

To further compare the sensitivity of SLR and LR for measuring a range of DNA damage, we subjected naked nDNA and mtDNA (extracted DNA in a small volume of Tris-EDTA buffer) to UVC. DNA damage generated by $5 \mathrm{~J} / \mathrm{m}^{2}\left(0.5 \mathrm{~mJ} / \mathrm{cm}^{2}\right)$ UVC should be in the range of $1.5-2$ lesions/10 kb [4], and this is what we measured by both

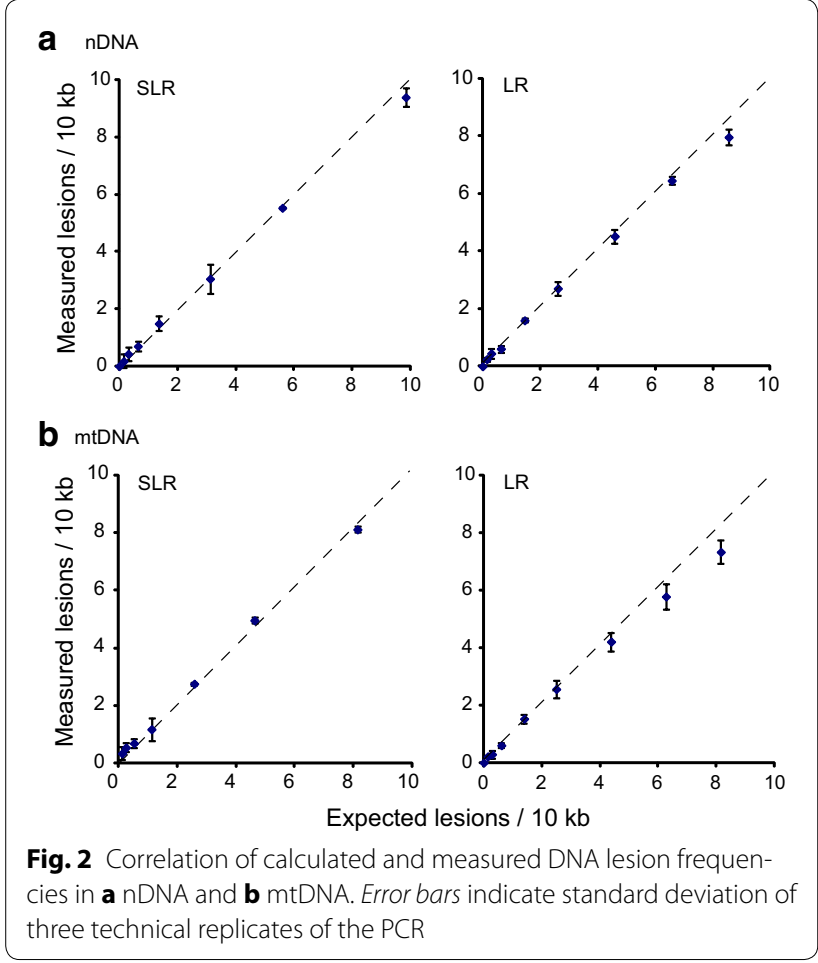

methods (Fig. 3; Additional file 1: Table S2). The results also showed that DNA damage increases in a UV dosedependent manner. At low dose of UVC, the amount of damage in both nDNA and mtDNA detected by SLR PCR is very close to that detected by LR PCR, indicating similar sensitivities. With increasing UVC dose, the signal produced by LR PCR saturated sooner than that produced by SLR PCR (Fig. 3), again indicating that SLR PCR is more sensitive than LR PCR at detecting higher levels of DNA damage.

\section{Use of SLR rtPCR to measure damage in nuclear and mitochondrial DNA from tissues of zebrafish of different ages}

As an application we asked whether SLR rtPCR could detect differences in the amount of DNA damage in the nuclear and/or mitochondrial genomes of different tissues of adult zebrafish of different ages, from young adulthood (5 months) through middle age (20 months). Nuclear DNA damage in all the four tissues of zebrafishes was found to increase with age, becoming statistically significant after 12-months (except in 20-month old heart sample) (Fig. 4a). Mitochondrial DNA damage increases with age in brain and liver, with the increase becoming statistically significant at 20-months in brain and after

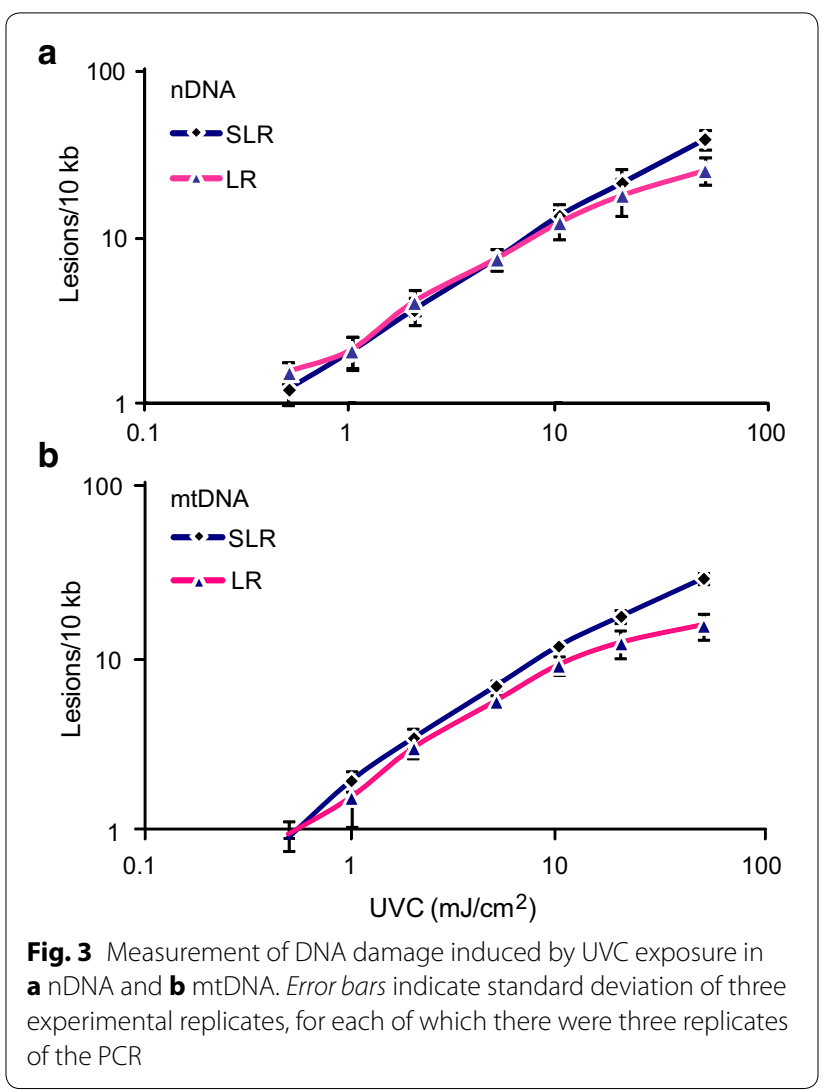




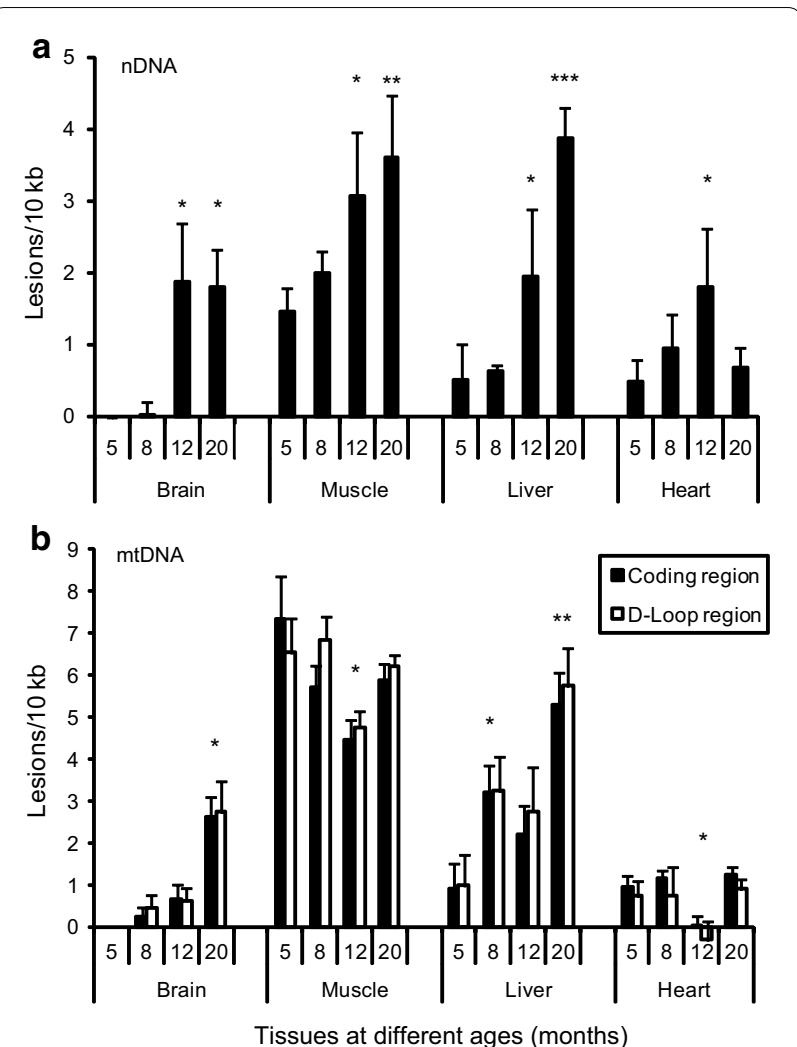

Fig. 4 DNA damage measured within a nuclear and $\mathbf{b}$ mitochondrial DNA from different tissues of zebrafish of different ages. Error bars indicate standard deviation of three replicate measurements from the same DNA, for each of which there were three replicates of the PCR. Significance (in comparison to 5 months using a two-tailed t test): ${ }^{*} p<0.05 ;{ }^{* *} p<0.01$; ${ }^{* * *} p<0.001$

8-months in liver. No significant change with age was observed in muscle and heart, except in the 12-month old heart sample which had less damage (Fig. 4b). Previous studies have shown that D-loop region of mtDNA, which exhibits a triple-stranded, semi-stable DNA structure during replication, is more vulnerable to oxidative damage and radiation $[5,6]$. We therefore asked whether the coding region and the control region containing the D-Loop structure had different amounts of DNA damage. Two-way ANOVA of the results did not show any significant difference in the cumulative in vivo damage displayed by the two regions of mtDNA in brain, muscle and liver, whereas the coding region had slightly higher levels of damage than D-Loop region $(\mathrm{p}<0.05)$ in heart.

\section{Discussion}

Our results indicate that SLR rtPCR provides several advantages to LORD-Q for quantifying DNA damage. With low levels of DNA damage, the sensitivity of SLR rtPCR is comparable to that of the LR rtPCR employed in
LORD-Q, whereas with high levels of DNA damage the sensitivity and hence accuracy of SLR rtPCR exceeds that of LORD-Q (Figs. 2, 3). This may be because the LR PCR generates more non-specific product (Fig. 1). In addition, the long amplicons utilized by LORD-Q are inherently less sensitive to high levels of DNA damage than are shorter amplicons: since a single lesion within the amplified region is likely sufficient to block amplification, multiple lesions within a single stretch of DNA are invisible to this method, leading to earlier signal saturation. While shorter amplicons are inherently more sensitive to higher levels of damage, they are less sensitive to low levels. Nevertheless, our results (Figs. 2, 3) demonstrate that SLR provides sensitivity equivalent to LORD-Q for detecting low levels of DNA damage. Since results from previous studies indicate that even shorter amplicons are less sensitive [5], we believe that the $\sim 2 \mathrm{~kb}$ employed by our SLR rtPCR method may be optimal for this type of analysis. Importantly, SLR rtPCR makes PCR optimization easier and hence faster, and offers greater flexibility in selection of fluorescent dyes for real time detection (Fig. 1). Finally, whereas the LORD-Q data analysis method requires the use of the Fit Point method to generate the amplification efficiency for the calculation of lesion frequency, our calculation of DNA lesion frequency using normalized concentration values enables the use of the 2nd derivative maximum analysis method (see "Methods"; Additional file 1: Table S3), which offers the advantages of higher accuracy, speed and simplicity.

Applying our method to both the nuclear and mitochondrial genomes of various somatic tissues in aging zebrafish we find that DNA damage accumulates with age, consistent with the widely held view that aging in senescing animals entails loss of genomic stability in somatic tissues. It should be noted that the average levels of DNA damage detected do not reflect per-cell levels of damage, but rather the average level of DNA damage throughout the tissue. Thus the increase in DNA damage seen in several of the tissues probably reflects increasing numbers of senescent or dying cells, rather than increasing loads of DNA damage within healthy cells. Although previous studies have examined the levels of DNA damage and repair capacities in specific tissues at different life cycle stages [7-11], none so far have reported direct comparisons of the amount of DNA damage in different tissues in an adult animal and its change with age. Our measurements showed that both brain and heart have lower nuclear and mitochondrial DNA damage than muscle and liver, consistent with the higher repair capacity that might be expected in the former tissues. The results from muscle and liver are consistent with studies showing that in most tissues levels of DNA damage are higher in mitochondria than in nuclei $[11,12]$. Finally, 
whereas damage in nuclear DNA was found to increase with age in each of the somatic tissues examined, damage in mitochondrial DNA increased in brain and liver, but not in heart or skeletal muscle, wherein the highest levels of DNA damage were found irrespective of age. These results suggest that aging entails increasing levels of nuclear DNA damage in somatic tissues, consistent with what has been found in previous studies $[13,14]$.

\section{Additional file}

Additional file 1. Additional tables.

\section{Abbreviations}

PCR: polymerase chain reaction; rtPCR: real-time PCR; LR: long run; SLR: semilong run; LORD-Q: long-run DNA damage quantification; nDNA: nuclear DNA; mtDNA: mitochondrial DNA.

\section{Authors' contributions}

SZ performed all the experiments and data analysis and drafted the figures and parts of the manuscript; JAC directed the research and wrote the manuscript. Both authors read and approved the final manuscript.

\section{Acknowledgements}

We thank Ellen I. Hartig for providing the zebrafish tissues used for DNA damage analysis.

\section{Competing interests}

The authors declare that they have no competing interests.

\section{Availability of data and materials}

All data generated or analysed during this study are included in this published article and its additional information files.

\section{Ethics approval and consent to participate}

All procedures involving vertebrate animals (zebrafish) were approved by the Institutional Animal Care and Use Committee (IACUC) of the MDI Biological Laboratory.

\section{Funding}

Research reported in this publication was supported by Institutional Development Awards (IDeA) from the National Institute of General Medical Sciences of the National Institutes of Health under Grant Numbers P20-GM104318 and P20-GM103423.

\section{Publisher's Note}

Springer Nature remains neutral with regard to jurisdictional claims in published maps and institutional affiliations.

Received: 30 December 2016 Accepted: 1 July 2017

Published online: 11 July 2017
References

1. Hunter SE, Jung D, Di Giulio RT, Meyer JN. The QPCR assay for analysis of mitochondrial DNA damage, repair, and relative copy number. Methods. 2010;51(4):444-51.

2. Santos JH, Meyer JN, Mandavilli BS, Van Houten B. Quantitative PCR-based measurement of nuclear and mitochondrial DNA damage and repair in mammalian cells. Methods Mol Biol. 2006:314:183-99.

3. Lehle S, Hildebrand DG, Merz B, Malak PN, Becker MS, Schmezer P, Essmann F, Schulze-Osthoff K, Rothfuss O. LORD-Q: a long-run real-time PCR-based DNA-damage quantification method for nuclear and mitochondrial genome analysis. Nucleic Acids Res. 2014;42(6):e41.

4. Meyer JN. QPCR: a tool for analysis of mitochondrial and nuclear DNA damage in ecotoxicology. Ecotoxicology. 2010;19(4):804-11.

5. Rothfuss O, Gasser T, Patenge N. Analysis of differential DNA damage in the mitochondrial genome employing a semi-long run real-time PCR approach. Nucleic Acids Res. 2010;38(4):e24

6. Zhou X, Liu X, Zhang X, Zhou R, He Y, Li Q, Wang Z, Zhang H. Non-randomized mtDNA damage after ionizing radiation via charge transport. Sci Rep. 2012;2:780.

7. Mitchell DL, Hartman PS. The regulation of DNA repair during development. BioEssays. 1990;12(2):74-9.

8. Barbin A, Ohgaki H, Nakamura J, Kurrer M, Kleihues P, Swenberg JA Endogenous deoxyribonucleic acid (DNA) damage in human tissues: a comparison of ethenobases with aldehydic DNA lesions. Cancer Epidemiol Biomark Prev. 2003;12(11 Pt 1):1241-7.

9. Karahalil B, Hogue BA, de Souza-Pinto NC, Bohr VA. Base excision repair capacity in mitochondria and nuclei: tissue-specific variations. FASEB J. 2002;16(14):1895-902.

10. Lin LH, Cao S, Yu L, Cui J, Hamilton WJ, Liu PK. Up-regulation of base excision repair activity for 8-hydroxy-2'-deoxyguanosine in the mouse brain after forebrain ischemia-reperfusion. J Neurochem. 2000;74(3):1098-105.

11. Hamilton ML, Guo Z, Fuller CD, Van Remmen H, Ward WF, Austad $\mathrm{SN}$, Troyer DA, Thompson I, Richardson A. A reliable assessment of 8-oxo-2-deoxyguanosine levels in nuclear and mitochondrial DNA using the sodium iodide method to isolate DNA. Nucleic Acids Res. 2001;29(10):2117-26.

12. Yakes FM, Van Houten B. Mitochondrial DNA damage is more extensive and persists longer than nuclear DNA damage in human cells following oxidative stress. Proc Natl Acad Sci USA. 1997;94(2):514-9.

13. Hamilton ML, Van Remmen H, Drake JA, Yang H, Guo ZM, Kewitt K, Walter CA, Richardson A. Does oxidative damage to DNA increase with age? Proc Natl Acad Sci USA. 2001;98(18):10469-74.

14. Best BP. Nuclear DNA damage as a direct cause of aging. Rejuvenation Res. 2009;12(3):199-208.

Submit your next manuscript to BioMed Central and we will help you at every step:

- We accept pre-submission inquiries

- Our selector tool helps you to find the most relevant journal

- We provide round the clock customer support

- Convenient online submission

- Thorough peer review

- Inclusion in PubMed and all major indexing services

- Maximum visibility for your research

Submit your manuscript at www.biomedcentral.com/submit 